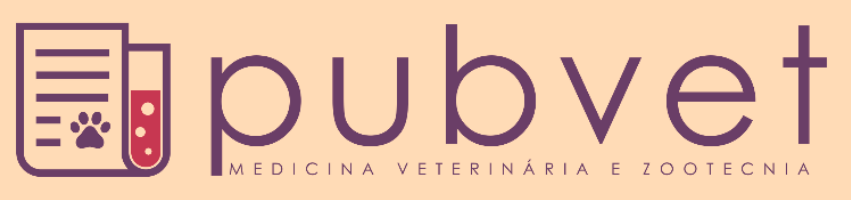

https://doi.org/10.31533/pubvet.v14n9a649.1-7

\title{
Diabetes mellitus em cães: buscando uma relação entre obesidade e hiperglicemia
}

\author{
Beatriz Pinheiro Amato ${ }^{190}$, Teresa Cristina Barros ${ }^{2 * 0}$ \\ ${ }^{I}$ Acadêmica de Medicina Veterinária, Universidade Paulista - UNIP - Bauru - SP. \\ ${ }^{2}$ Química - Doutora em Bioquímica - Professora da Universidade Paulista - UNIP - Bauru - SP. \\ *Autor para correspondência, E-mail: tcbarr@uol.com.br
}

\begin{abstract}
Resumo. Estudos sobre Diabetes Mellitus em cães são de extrema importância, já que conhecimentos mais aprofundados possibilitam que a profilaxia da enfermidade possa ser colocada em prática, além de fazer com que os diagnósticos sejam mais rápidos havendo, consequentemente, tratamentos corretos e com melhores resultados. Além disso, a disseminação de informações pode diminuir o número de casos em longo prazo. Sendo assim, o objetivo deste trabalho foi avaliar a existência ou não da relação entre hiperglicemia e obesidade em cães. Foram distribuídos panfletos informativos, disponibilizando alguns dias para que proprietários de cães levassem seus animais, gratuitamente, até a clínica veterinária da UNIP - Campus Bauru, para que fosse feita uma análise dos seus animais. Estes proprietários levaram seus animais com um jejum de no mínimo 8 horas e responderam algumas questões relativas à alimentação e atividade física de seus cães. Foram utilizados parâmetros complementares para caracterizar obesidade, mas, mesmo assim, os animais não apresentaram relação entre obesidade e hiperglicemia. De posse de dados da literatura mais recentes, verificou-se que os resultados obtidos estão de acordo com uma diferente etiologia para o desenvolvimento de diabetes em cães, e que não está relacionado com a obesidade e resistência à insulina.
\end{abstract}

Palavras-chaves: caninos, endocrinologia, sobrepeso

\section{Diabetes mellitus in dogs: searching a relationship between obesity and hyperglycemia}

\begin{abstract}
Studies on Diabetes Mellitus in dogs are of extreme importance, since a more profound understanding allow the prophylaxis of disease can be put into practice. In that way, as a consequence, the treatments would have better results. In addition, the dissemination of information can lower down the numbers of cases in a long term. Besides that, the objective of this article was to evaluate the existence or not of the relation between hyperglycemia and obesity in dogs. Informative leaflets were distributed, providing some days for owners of dogs take their animals, for free, to the veterinary clinic of the UNIP Campus of Bauru, for it was made an analysis of their animals. These owners took their animals without a fasting for at least 8 hours and answered some questions relating to nutrition and physical activity of their dogs. Complementary parameters were used to characterize obesity, but even so, the animals showed no relationship between obesity and hyperglycemia. In possession of more recent data from the literature, we verified that the results obtained were in agreement with a different etiology for the development of diabetes in dogs, and that is not related to obesity and insulin resistance.
\end{abstract}

Keywords: canine, endocrinology, overweight 


\section{Introdução}

A Diabetes Melittus, nome escolhido para diferenciar da diabetes insípida, a qual é uma deficiência de vasopressina $(\mathrm{ADH})$ e sem qualquer relação com a concentração sanguínea de glicose, é uma endocrinopatia crônica sistêmica que vem aparecendo cada vez mais no cotidiano das clínicas veterinárias. A proporção de animais com a doença seria de $1 \mathrm{em}$ cada 60 cães, sendo predominante em fêmeas, idosas (com mais de 7 anos) e obesas (Faria, 2007; Gonzalez \& Silva, 2006).

As células beta $(\beta)$ pancreáticas produzem o hormônio insulina, o qual participa na homeostase glicêmica, sendo secretada logo após a ingestão de alimentos contendo glicose e aminoácidos e tem seus valores em menores quantidades em momentos de jejum. A insulina ativa a captação de glicose em algumas células (células insulino dependentes, como as musculares e adiposas), sendo ainda responsável por ativar a síntese da reserva de carboidratos (glicogênio) e de lipídeos (triacilglicerol), e inibir a lise de gordura estocada no tecido adiposo (Cunningham, 2014; Faria, 2007).

A Diabetes Melittus em cães é tão perigosa quanto a Diabetes em humanos. Assim, estudos sobre a doença são de grande valia para que haja um conhecimento mais aprofundado sobre ela, de forma que a profilaxia da doença pode ser colocada em prática (quando possível), fazendo com que diagnósticos sejam mais rápidos e, consequentemente, havendo tratamentos corretos e com melhores resultados.

Além disso, a disseminação de informações sobre a enfermidade, sua profilaxia e a importância dos cuidados, deve conscientizar a população e gerar uma diminuição no número de casos em longo prazo.

Embora a Diabetes Melittus tipo 1 (DM1), onde há deficiência na produção de insulina, seja o tipo mais comumente encontrado em cães, a proposta deste trabalho foi verificar uma possível relação entre a obesidade canina e o desenvolvimento de Diabetes Melittus tipo 2 (DM2), onde ocorre resistência à ação da insulina. Para isso foram utilizados diferentes parâmetros para identificar um cão como sendo obeso ou não, além da relação entre o seu peso e estatura, o que indica o índice de massa corporal canino (IMCC).

\section{Materiais e Métodos}

Foram avaliados 10 animais que se apresentavam com no mínimo 8 horas de jejum. Antes da coleta do material biológico, foram obtidas informações sobre alimentação, castração e atividades físicas exercidas pelo animal. Em seguida, foram analisados alguns dados antropométricos, como altura, morfometria e peso de cães.

A porcentagem de gordura corporal (\%GC), foi obtida seguindo o método citado por Mawby et al. (2004). Para isso, foram realizadas medidas do membro pélvico (MP) e do perímetro abdominal (PA) com o auxílio de uma fita métrica simples. Os valores foram usados para os seguintes cálculos:

Fêmea \%GC $=-1,7 \times(\mathrm{MPcm})+0,93 \times(\mathrm{PAcm})+5$

Machos $\% \mathrm{GC}=-1.4 \mathrm{x}(\mathrm{MPcm})+0.77(\mathrm{PAcm})+4$

Sendo:

$\mathrm{MP}=\mathrm{o}$ comprimento entre a tuberosidade do calcâneo e o ligamento patelar médio, externamente.

$\mathrm{PA}=$ ponto médio entre a asa do íleo e a última vértebra torácica

Para o Índice de Massa Corporal Canino (IMCC), foi utilizada uma adaptação do IMC humano:

IMCC = peso corporal $(\mathrm{kg}) /$ estatura $^{2}\left(\mathrm{~m}^{2}\right)$

Os pontos de referência para a determinação de estatura são o comprimento da articulação atlantooccipital até o solo, apoiando a fita sobre a última vértebra sacral e passando atrás dos membros posteriores (Muller, Schossler, \& Pinheiro, 2008).

Caso o IMC tenha um valor inferior a 18,5 o animal está abaixo do peso. Os valores que representam o índice de normalidade estão entre 18,5 e 25 . IMC entre 25 e 30 estão relacionados ao sobrepeso e valores superiores a 30 sugerem obesidade (Muller et al., 2008). 
A análise da glicemia foi realizada com uso de glicosímetro digital a partir de perfuração na orelha ou na pata dos animais. Os valores de referência (Pöppl \& González, 2005) para glicemia canina são:

Normal: 60 a $120 \mathrm{mg} / \mathrm{dL}$

Hipoglicemia: menor que $60 \mathrm{mg} / \mathrm{dL}$

Hiperglicemia: maior que $120 \mathrm{mg} / \mathrm{dL}$

Além disso, foi realizada a classificação de ECC (Escore Corporal Canino), descrito por Laflamme (2006), a qual se refere à condição corporal em que o animal se enquadra, através de sua observação, classificando o animal de 1 a 9, ou seja, subalimentado a sobrealimentado (Tabela 1).

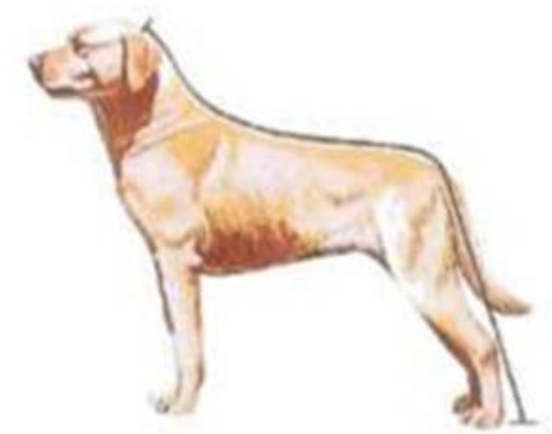

Figura 1. Trajeto para obtenção do IMCC delimitada pela linha preta (uller et al. 2008).

Tabela 1. Medida de Escore Corporal Canino (ECC).

\begin{tabular}{|c|c|c|}
\hline Condição Corporal & $\begin{array}{c}\text { Escore } \\
\text { Corporal }\end{array}$ & Características da Condição Corporal \\
\hline \multirow{3}{*}{ Subalimentado } & 1 & $\begin{array}{l}\text { Não há gordura corporal perceptível e há perda evidente de massa muscular. } \\
\text { As costelas, vértebras lombares, ossos pélvicos e todas as saliências ósseas são } \\
\text { visíveis à distância. }\end{array}$ \\
\hline & 2 & $\begin{array}{l}\text { Costelas, vértebras lombares, ossos pélvicos com fácil visualização. } \\
\text { Gordura não palpável. } \\
\text { Pode haver algumas outras saliências ósseas visíveis. } \\
\text { Há perda mínima de massa muscular. }\end{array}$ \\
\hline & 3 & $\begin{array}{l}\text { Costelas facilmente palpáveis podem estar visíveis, porém sem gordura papável. } \\
\text { Topo das vertebras lombares visíveis. } \\
\text { A cintura e reentrância abdominal são evidentes. }\end{array}$ \\
\hline \multirow{3}{*}{ Ideal } & 4 & $\begin{array}{l}\text { As costelas são facilmente palpáveis e possuem mínima cobertura de gordura. } \\
\text { A cintura é facilmente observada quando vista de cima. } \\
\text { Há reentrância abdominal evidente. }\end{array}$ \\
\hline & 5 & $\begin{array}{l}\text { O abdome fica retraído quando visto de lado. } \\
\text { As costelas são palpáveis e não possuem excessiva cobertura de gordura. }\end{array}$ \\
\hline & 6 & $\begin{array}{l}\text { Costelas são palpáveis com pouco excesso de cobertura de gordura. } \\
\text { Quando vista de cima, a cintura é visível, porém ela não é acentuada. } \\
\text { A reentrância abdominal é aparente. }\end{array}$ \\
\hline \multirow{3}{*}{ Sobrealimentado } & 7 & $\begin{array}{l}\text { Dificuldade na palpação das costelas, pois há intensa cobertura de gordura. } \\
\text { Presença de depósitos de gordura evidentes acima da área lombar e base da cauda } \\
\text { Cintura apenas visível ou ausente. } \\
\text { Reentrância abdominal pode estar presente. }\end{array}$ \\
\hline & 8 & $\begin{array}{l}\text { Cintura e reentrância abdominal inexistentes. } \\
\text { Pode haver distensão abdominal evidente. } \\
\text { As costelas estão situadas sob uma cobertura muito densa de gordura, portanto } \\
\text { impossível sua palpação (são palpáveis apenas com pressão acentuada). }\end{array}$ \\
\hline & 9 & $\begin{array}{l}\text { Grandes depósitos de gordura sobre o tórax, espinha e base da cauda, assim como } \\
\text { também há deposito de gordura no pescoço e membros. } \\
\text { A distensão abdominal é evidente. }\end{array}$ \\
\hline
\end{tabular}

Fonte: Adaptado de Laflamme (2006). 


\section{Resultados}

A amostra avaliada foi de 10 cães, sendo 9 fêmeas e 1 macho, cuja média de idade equivale a 7 anos (Tabela 2).

Tabela 2. Parâmetros obtidos através do questionário

\begin{tabular}{ccccccccc}
\hline No & Idade & Sexo & Raça & Castrado & Alimentação* & Vezes Oferecidas & Ativ./Semana & Jardim/Quintal \\
\hline 1 & 8 anos & Fêmea & Labrador & Sim & 1 & 2 & Raro & Sim \\
2 & 11 anos & Fêmea & Lhasa Apso & Sim & 1 & Mais de 2 & 2 ou mais & Não \\
3 & 12 anos & Fêmea & Lhasa Apso & Não & 1 & 2 & Não & Sim \\
4 & 8 anos & Fêmea & Lhasa Apso & Não & 1 & 2 & Não & Sim \\
5 & 3 anos & Fêmea & Beagle & Sim & 2 & Mais de 2 & 2 ou mais & Sim \\
6 & 7 meses & Fêmea & Poodle Toy & Não & 4 & Mais de 2 & Todos os dias & Sim \\
7 & 12 anos & Fêmea & Akita & Sim & 4 & 2 & Todos os dias & Sim \\
8 & 13 anos & Fêmea & Poodle & Não & 4 & 2 & Não & Sim \\
9 & 7 anos & Fêmea & Poodle Toy & Sim & 1 & Mais de 2 & Não & Sim \\
10 & 4 anos & Macho & Poodle Toy & Sim & 1 & Mais de 2 & Não & Sim \\
\hline
\end{tabular}

*1 - Apenas ração. 2 - Comida caseira/restos de comida e ração. 3 - Comida caseira/restos de comida, 4 - Ração e petiscos

Foram obtidos os pesos destes animais, bem como determinados os parâmetros de IMCC, ECC, \% GC e glicemia em jejum (pelo menos 8 horas de jejum). Todos os dados obtidos, para cada animal, estão apresentados, a seguir (Tabela 3). Observa-se que 60\% dos aninais têm pouca atividade física e, destes, $50 \%$ são castrados.

Tabela 3. Parâmetros obtidos pela avaliação dos animais.

\begin{tabular}{lccccccc}
\hline $\mathrm{N}^{\circ}$ & Sexo & Raça & Peso, Kg & ECC & $\%$ CG & Glicemia & IMCC, $\mathrm{kg} / \mathrm{m}^{2}$ \\
\hline 1 & Fêmea & Labrador & 37,0 & 9 & 45,85 & 94 & 22,94 \\
2 & Fêmea & Lhasa Abso & 9,5 & 8 & 37,13 & 92 & 23,2 \\
3 & Fêmea & Lhasa Abso & 4,1 & 3 & 16,83 & 50 & 11,78 \\
4 & Fêmea & Lhasa Abso & 6,3 & 5 & 19,94 & 70 & 14,91 \\
5 & Fêmea & Beagle & 23,4 & 7 & 33,9 & 86 & 30,21 \\
6 & Fêmea & Podle Toy & 2,8 & 4 & 8,18 & 90 & 7,8 \\
7 & Fêmea & Akita & 85,0 & 9 & 51,91 & 54 & 43,4 \\
8 & Fêmea & Podle & 6,9 & 4 & 10,2 & 64 & 11,94 \\
9 & Fêmea & Podle Toy & 4,0 & 8 & 21,32 & 83 & 14,8 \\
10 & Macho & Podle Toy & 6,0 & 7 & 26,74 & 80 & 19,83 \\
\hline
\end{tabular}

O peso obtido para cada animal foi comparado com dados da literatura, de acordo com cada raça, verificando-se que, dentre os cães analisados, apenas 4 deles (40\%) não estão acima do peso (os cães de número 3, 4, 6 e 8). A Tabela 4 mostra aproximadamente qual seria o peso ideal de cada raça dos animais que participaram desta amostra.

Tabela 4. Peso ideal para cães de acordo com as suas raças

\begin{tabular}{lcc}
\hline Raça & Peso Macho, kg & Peso Fêmea, kg \\
\hline Akita & 34,0 & 34,0 \\
Beagle & 6,0 a 10,0 & 6,0 a 9,0 \\
Labrador & 29,0 a 36,0 & 25,0 a 31,0 \\
Lhasa Apso & 7,0 & 6,0 \\
Poodle & 9,0 a 13,5 & 9,0 a 13,5 \\
Poodle Toy & 3,1 a 4,5 & 3,1 a 4,5 \\
\hline
\end{tabular}

Fonte: Adaptado de Rodrigues (2011).

A relação entre a porcentagem de Gordura Corporal (\%CG) e o Escore Corporal Canino (ECC), um dos parâmetros utilizados nas análises dos animais, está apresentada na Tabela 5. 
Considerando-se os parâmetros de IMCC, ECC e \%GC e peso ideal para cada uma das raças, os animais 1, 2, 4, 5, 7 e 10 devem ser considerados obesos, ou seja, 60\% dos animais avaliados foram considerados obesos (com aumento de $20 \%$ de seu peso ideal). No entanto, como verificado na Tabela $\underline{3}$, não houve relação entre hiperglicemia e obesidade, já que nenhum destes animais apresentou glicemia elevada.

Tabela 5. Gordura corporal máxima de acordo com cada faixa de ECC

\begin{tabular}{ccc}
\hline ECC & \% Machos & \% Fêmeas \\
\hline 5 & 17 & 20 \\
6 & 22 & 26 \\
7 & 26 & 31 \\
8 & 31 & 37 \\
9 & $35+$ & $43+$ \\
\hline
\end{tabular}

Fonte: Adaptado de Laflamme (2006).

Posteriormente, os proprietários foram contatos através de seus e-mails, onde lhes foi enviado o resultado da análise de glicemia, IMCC e porcentagem de gordura corporal (\%GC), acompanhado com a explicação da situação do animal.

Assim, os resultados deste trabalho indicaram que, mesmo utilizando parâmetros mais completos para identificar a obesidade canina, estes cães considerados obesos não apresentam DM2.

\section{Discussão}

Sabe-se que a diabetes mellitus tipo I (insulino dependente) é a forma mais comumente encontrada em cães e possui caráter multifatorial. Alguns fatores são potencialmente predisponentes como predisposição genéticas, lesões pancreáticas (pancreatites redicivantes ou neoplasias) e componentes imunomediados, podendo ser através de insulite imunomediada com infiltração de linfócitos nas ilhotas pancreáticas, além de anticorpos contra as células da ilhota, pró-insulina, insulina, ácido glutamico descarboxilase intracelular e antígeno de insulinoma 2. Esses componentes fazem com que a insulina esteja ausente ou responda de forma insuficiente, gerando assim um quadro de hiperglicemia (Nelson, 1992; Nelson \& Reusch, 2014).

Existem relatos de que 46\% (quarenta e seis por cento) dos cães com DM1 apresentam infiltração inflamatória nas ilhotas pancreáticas e soro toxicidade contra as células beta. Além disso, aproximadamente $50 \%$ (cinquenta por cento) dos cães diabéticos apresentam anticorpos contra a insulina ou um antígeno da membrana das células beta (Fleeman \& Rand, 2001; Rand, Fleeman, Farrow, Appleton, \& Lederer, 2004).

As fêmeas não castradas têm maior propensão a terem diabetes, pois o estrógeno e a progesterona diminuem a sensibilidade à insulina nos órgãos-alvos. Alguns fármacos, como glicocorticoides e progestágenos sintéticos estão associados como causas iatrogênicas da doença, pois sabe-se que a terapia prolongada com corticoide reduz a função das células beta, e, consequentemente, gera processos imunológicos ou pancreatite crônica, além de altos níveis de progesterona causarem intolerância a glicose, como dito anteriormente (Faria, 2007; Rand et al., 2004).

A predisposição genética é frequente em algumas raças como Malamute do Alaska, Golden Retriever, Keeshounds, Spitz, Whippet, Pulik, Cairn Terrier, Schanauzer, Pinscher Miniatura e SRDs (sem raça definida), embora perceba-se que o stress e a idade estejam aumentando cada vez mais o número de animais doentes (Faria, 2007).

A hiperlipidemia, ou seja, aumento do catabolismo de lipídio de reserva como fonte de energia, com aumento de ácidos graxos livres na corrente sanguínea, insulite imunomediada e amiloidose nas ilhotas pancreáticas, são alguns outros fatores importantes para o desenvolvimento de diabetes (Gonzalez \& Silva, 2006; Pöppl \& González, 2005).

A obesidade, causada por alimentação rica em gordura e pobre em carboidrato, ocasiona redução dos efeitos de insulina no sistema nervoso central, o que leva ao estímulo de fome e inibição do centro de 
saciedade do hipotálamo. Consequentemente, tem-se um aumento de ácidos graxos livres, que reduzem a fosforilação de mensageiros intracelulares causando menor resposta à insulina diminuindo assim, a sensibilidade dos tecidos periféricos ao hormônio. Como resultado, haverá uma hiperinsulinemia e diferentes graus de intolerância a glicose (Gonzalez \& Silva, 2006).

Uma causa que relaciona a obesidade e a diabetes é que o aumento de peso diminui a expressão gênica e proteica do transportador de glicose GLUT 4 na membrana das células musculares e de tecidos adiposos. Além disso, um cão obeso precisa de maior aporte de glicose para se manter e o constante estímulo para liberação de insulina pelo pâncreas irá gerar exaustão das células beta em médio a longo prazo, diminuindo a produção de insulina (Veiga, 2018).

Segundo Nelson \& Reusch (2014), cães obesos podem apresentar resistência ao hormônio insulina, mas não há progressão para diabetes tipo 2 , já que não existe mecanismos etiopatogênicos, presentes em humanos e felinos, responsáveis pelo seu desenvolvimento.

Assim, muitos dados na literatura apontam para a não equivalência entre diabetes em humanos e em cães, sendo que a diabetes em cães não estaria associada à resistência à insulina, mas sim a fatores genéticos ou outros que levem a uma falta ou ineficiência na produção de insulina (DM1) (Pöppl \& González, 2005).

Em geral, a hiperglicemia observada em humanos diabéticos e obesos está associada, dentre outros fatores, a um processo inflamatório provocado pelos ácidos graxos livres que estão em excesso na corrente sanguínea, como resultado do maior volume do tecido adiposo. Esta situação pode gerar uma resistência à insulina, com o objetivo de garantir maior disponibilidade de glicose para o cérebro (McLellan, Manda, Sloan, \& Burini, 2013; Schelp \& Burini, 1995). Neste caso, pessoas obesas tendem a garantir a não absorção de glicose pelos grandes tecidos, a fim de manter a atividade do seu sistema nervoso central. Porém, esta situação não acontece, necessariamente, de maneira similar em animais, visto que não utilizam concentração tão elevadas de glicose para atividade cerebral.

Quanto maior o quociente cerebral, ou seja, a relação entre massa do cérebro e massa corporal, maior será a necessidade energética do cérebro. Embora não seja aqui apresentada a comparação entre os quocientes cerebrais de homens e cães, o córtex dos cães possui $65,5 \mathrm{~g}$, enquanto que de um ser humano chega a ter 233,68g. Além disso, os homens possuem mais neurônios: são 86 bilhões (homem de 60 70 anos) comparados aos 500 milhões dos caninos. Isso está relacionado com uma atividade cerebral mais evoluída em relação ao raciocínio e inteligência, tornando o consumo de glicose diária maior para os humanos. (Jardim-Messeder et al., 2017; Lent, Azevedo, Andrade-Moraes, \& Pinto, 2012).

Cabe lembrar que esta comparação de necessidade de glicose para o funcionamento cerebral não é válida ao ser discutida a DM2 em gatos, visto que estes animais apresentam, naturalmente, níveis mais altos de glicose sanguínea pela difícil digestão e absorção de carboidratos, bem como por serem animais considerados neoglicogênicos (Carciofi, 2007).

\section{Conclusão}

Feita a análise dos dados, concluiu-se que nenhum dos animais, mesmo aqueles considerados obesos utilizando-se outros parâmetros, além do IMCC, apresentaram hiperglicemia ou diabetes. Esse fato condiz com o que é relatado em literatura, ou seja, que a obesidade está associada diretamente apenas com a fisiopatologia da diabetes tipo II de felinos e humanos, mas não na espécie canina.

\section{Referências bibliográficas}

Carciofi, A. C. (2007). Métodos para estudo das respostas metabólicas de cães e gatos a diferentes alimentos. Revista Brasileira de Zootecnia, 36(suppl), 235-249. https://doi.org/10.1590/S1516$\underline{35982007001000022}$

Cunningham, J. (2014). Tratado de fisiologia veterinária (Elsevier). Rio de Janeiro: Guanabara Koogan.

Faria, P. F. (2007). Diabetes mellitus em cães. Acta Veterinária Brasílica, 1(1), 8-22. https://doi.org/https://doi.org/10.21708/avb.2007.1.1.258

Fleeman, L. M., \& Rand, J. S. (2001). Management of Canine Diabetes. Veterinary Clinics of North 
America: Small Animal Practice, 31(5), 855-880. https://doi.org/10.1016/S0195-5616(01)50003-0

Gonzalez, F. H. D., \& Silva, S. C. (2006). Introdução à bioquímica clínica animal. Porto Alegre, Rio Grade do Sul: Gráfica de Universidade Federal do Rio Grande do Sul.

Jardim-Messeder, D., Lambert, K., Noctor, S., Pestana, F. M., Castro Leal, M. E., Bertelsen, M. F., Herculano-Houzel, S. (2017). Dogs Have the Most Neurons, Though Not the Largest Brain: TradeOff between Body Mass and Number of Neurons in the Cerebral Cortex of Large Carnivoran Species. Frontiers in Neuroanatomy, 11, 118. https://doi.org/10.3389/fnana.2017.00118

Laflamme, D. P. (2006). Understanding and Managing Obesity in Dogs and Cats. Veterinary Clinics of North America: Small Animal Practice, 36(6), 1283-1295. https://doi.org/10.1016/j.cvsm.2006.08.005

Lent, R., Azevedo, F. A. C., Andrade-Moraes, C. H., \& Pinto, A. V. O. (2012). How many neurons do you have? Some dogmas of quantitative neuroscience under revision. European Journal of Neuroscience, 35(1), 1-9. https://doi.org/10.1111/j.1460-9568.2011.07923.x

Mawby, D. I., Bartges, J. W., D’Avignon, A., Laflamme, D. P., Moyers, T. D., \& Cottrell, T. (2004). Comparison of Various Methods for Estimating Body Fat in Dogs. Journal of the American Animal Hospital Association, 40(2), 109-114. https://doi.org/10.5326/0400109

McLellan, K. C. P., Manda, R. M., Sloan, L. A., \& Burini, R. C. (2013). Epigenetics of Glucose Metabolism and the Basis for T2DM Interventions. Type 2 Diabetes, 51.

Muller, D. C. M., Schossler, J. E., \& Pinheiro, M. (2008). Adaptação do índice de massa corporal humano para cães. Ciência Rural, 38(4), 1038-1043. https://doi.org/10.1590/S0103$\underline{84782008000400020}$

Nelson, R. W. (1992). Distúrbios do pâncreas endócrino. In: ETTINGER, SJ Tratado de Medicina Interna Veterinária (pp. 1752-98.). São Paulo: São Paulo: Editora Manole Ltda,.

Nelson, Richard W., \& Reusch, C. E. (2014). ANIMAL MODELS OF DISEASE: Classification and etiology of diabetes in dogs and cats. Journal of Endocrinology, 222(3), T1-T9. https://doi.org/10.1530/JOE-14-0202

Pöppl, Á. G., \& González, F. H. D. (2005). Aspectos epidemiológicos e clínico-laboratoriais da diabetes mellitus em cães. Acta Scientiae Veterinariae, 33(1), 33-40.

Rand, J. S., Fleeman, L. M., Farrow, H. A., Appleton, D. J., \& Lederer, R. (2004). Canine and Feline Diabetes Mellitus: Nature or Nurture? The Journal of Nutrition, 134(8), 2072S-2080S. https://doi.org/10.1093/jn/134.8.2072S

Rodrigues, L. F. (2011). Métodos de avaliação da condição corporal em cães. Universidade Federal de Goiás. Goiânia.

Schelp, A. O., \& Burini, R. C. (1995). Controle do fornecimento e da utilização de substratos energéticos no encéfalo. Arquivos de Neuro-Psiquiatria, 53(3b), 690-697. https://doi.org/10.1590/S0004$\underline{282 X 1995000400025}$

Veiga, A. P. M. (2018). Suscetibilidade a diabetes mellitus em cães obesos. Acta Scientiae Veterinariae, 36(3), 311. https://doi.org/10.22456/1679-9216.17310

Recebido: 9 de abril, 2020 .

Aprovado: 18 de maio, 2020.

Disponível online: 4 de setembro, 2020.

Licenciamento: Este artigo é publicado na modalidade Acesso Aberto sob a licença Creative Commons Atribuição 4.0 (CC-BY 4.0), a qual permite uso irrestrito, distribuição, reprodução em qualquer meio, desde que o autor e a fonte sejam devidamente creditados. 